\title{
Rapport général de la question III Deltas, estuaires et littoral
}

\author{
par L.A. Romaña \\ Ifremer, Centre de Toulon-La Seyne. \\ Direction de l'environnement et de l'aménagement littoral.
}

Le titre de ces Journées de I'Hydraulique «L'eau, l'homme et la nature» est de nature à nous inciter à développer et appliquer au littoral certaines grandes théories de cette fin de siècle sur les conséquences de l'action anthropique sur notre environnement. Afin de rester modestes, les organisateurs de ce colloque nous ont incité à mieux cibler les problèmes qui se posent sur le littoral, en nous demandant de les situer par rapport à une logique de continuité entre l'amont et l'aval, et principalement en suivant les «Chemins de l'eau, de la source à la mer...»

En suivant ce chemin, l'eau, à l'entrée des estuaires a déjà subi maintes transformations quantitatives et qualitatives. Elle a perdu des particules en suspension décantées dans les différents barrages et retenues ; elle s'est chargée en sels nutritifs et matière organique, conséquence des apports urbains et agricoles ; elle s'est parfois eutrophisée ; elle a recueilli pendant son parcours des contaminants chimiques d'origine industrielle ou domestique, des herbicides et des pesticides, des microorganismes tels que les virus, bactéries et autres parasites... Elle a vu le milieu se modifier progressivement, apparaître la diversité des espèces pélagiques et benthiques. Les aménagements, l'altération de la qualité des eaux et des sédiments a modifié les niches écologiques et a conduit à la désorganisation des chaînes trophiques.

Quelles seront les conséquences au niveau des estuaires, des deltas ou du littoral, de la modification du contenu biologique ou chimique de cette particule d'eau ? Les connaissances techniques et scientifiques actuelles sont-elles suffisantes pour permettre de faire cette évaluation? Sait-on vraiment quantifier les niveaux de contamination à ne pas dépasser, de façon à édicter des normes adaptées à la protection des organismes marins ou estuariens dont les premiers stades de vie sont très sensibles aux agressions de tous ordres...? Est-il possible de quantifier précisément les concentrations en contaminants que l'on trouvera dans ces différents milieux de façon à mieux contrôler les apports amont en vue d'améliorer l'assainissement ? Peut-on privilégier une démarche véritablement centrée sur la protection du milieu ?

Nombreux sont les exemples en France, et ailleurs, où les aménageurs n'ont pas toujours su prévoir, par manque de connaissances scientifiques, les conséquences parfois très en aval d'ouvrages réalisés en amont des cours d'eau. Un exemple déjà classique est offert par l'exemple des grands estuaires et deltas français. Situés à l'interface entre les continents et les océans, ils reçoivent les flux de contaminants issus en grande partie de l'activité agricole et indus- trielle, auxquels se rajoutent les rejets de la population des quatre grands bassins hydrologiques.

La conjonction entre la marée et le débit fluvial est à l'origine dans nos estuaires de la constitution d'un stock sédimentaire appelé bouchon vaseux et dont le mécanisme est décrit dans les communications de M. Sanchez et al. ainsi que dans celle de $\mathrm{P}$. Bassoullet et $\mathrm{P}$. Le Hir. Ce stock sédimentaire, très important, se déplace dans l'estuaire d'amont en aval au gré des débits amont. Il se dépose (crème de vase) ou se remet en suspension en fonction des cycles de marée (bi-journalier ou lunaire). Toute particule et, par conséquent tout élément associé à cette particule issue de l'amont (et parfois de la mer) séjournera dans cette zone pendant des périodes pouvant atteindre plusieurs années. Ce phénomène, déjà connu, est à l'origine d'une augmentation locale des concentrations en contaminants chimiques, de matière organique biodégradable, de germes pathogènes. Le cas de l'estuaire de la Loire avec une mortalité estivale de mulets en est un exemple.

Deux exemples d'application à la Loire seront présentés dans ce colloque : l'un s'attache à étudier le transit amontaval du matériel non cohésif (sables) en présence de la crème de vase (matériel cohésif); l'autre envisagera les conséquences de la remontée probable du niveau de la mer sur le fonctionnement hydrosédimentaire de cet estuaire.

Barrages et réservoirs ont considérablement réduit les transports solides vers l'aval, avec des conséquences que l'on commence seulement récemment à évaluer sur l'érosion littorale et la tenue des côtes... Nous aurons beaucoup d'exemples parmi les communications présentées dans ces journées : la Camargue, le Cotentin, le littoral Biarrot, la Baie de Seine, le littoral Ivoirien...

Certains de ces exposés s'attachent à établir un constat en proposant des solutions qui leur apparaissent les plus pertinentes. D'autres, à l'aide d'expériences sur le terrain, essayent de comprendre les mécanismes nécessaires à une bonne modélisation mathématique du transport sédimentaire. C'est ainsi que deux communications cherchent à mieux apprécier et quantifier les processus qui interviennent : approche expérimentale de la vitesse de chute des particules pour l'un, application de modèles 1 DV à des résultats expérimentaux en bassin pour les autres.

Enfin, à l'aide d'outils mathématiques développés à cet effet, des auteurs cherchent à reproduire l'hydrodynamique ou le transport sédimentaire d'une zone, de façon à apporter une meilleure réponse aux problèmes d'aménagement posés. Plusieurs cas sont étudiés : la baie de Monaco dans le cadre 
d'une étude financée par la communauté européenne, un système d'alerte contre les inondations pour la région de Shangaï, les effets de la houle sur le transport sédimentaire en baie et estuaire de Seine, l'aménagement du port d'Antifer, l'évolution morphologique d'une barrière littorale au Ghana.

Mais l'eau et les particules, à travers l'hydrodynamique et le transport sédimentaire véhiculent des contaminants chimiques et bactériens. La connaissance et la quantification des lois qui régissent la transformation de ces composés sont encore à leur début. Les premiers modèles mathématiques permettant de reproduire en milieu côtier le devenir de ces composés sont en cours de développement. Pour les composés chimiques, une contribution s'attache à décomposer et à évaluer les processus qui interviendront en milieu estuarien (encore la Loire) sur la géochimie du cadmium. Une dernière contribution, à caractère plus appliqué, tentera, à l'aide de différents outils mathématiques, de conseiller les responsables de l'assainissement dans la zone du Tréport, sur les dispositions à prendre pour améliorer la qualité des eaux de baignade de la région.

L'hydrodynamique et le transport sédimentaire étant essentiels pour la compréhension des phénomènes biologiques, une majorité de communications traitent de ces disciplines. Nous aborderons donc d'abord l'ensemble des communications qui s'y rattachent, celles concernant le milieu estuarien, puis celles traitant de l'érosion côtière. Avant de présenter quelques applications de ces outils mathématiques à des cas d'aménagement, nous passerons en revue deux communications sur des expériences menées en laboratoire et destinées à étudier certains processus sédimentologiques. Enfin nous traiterons de deux rapports portant sur l'utilisation des modèles mathématiques en qualité des eaux.

\section{D DYNAMIQUE SÉDIMENTAIRE EN MILIEU ESTUARIEN}

Première étape lors du passage de l'eau et des particules vers le milieu marin, les systèmes estuariens présentent un comportement hydrosédimentaire particulier, ce qui leur confère une spécificité propre. Son maximum de turbidité en vives eaux, sa crème de vase en mortes eaux, les temps de séjour plus importants pour les masses d'eau et les particules expliquent en grande partie ces caractéristiques.

Il faut aussi rappeler la variabilité naturelle extraordinaire de ces milieux, en particulier des paramètres essentiels au bon développement de la flore et de la faune : la turbulence, la lumière, la turbidité, la salinité, la température. Les siècles ont ainsi engendré des systèmes en équilibre instable, mais ce qui est le plus étonnant réside dans le fait qu'ils ne dépendent que de quelques paramètres hydrologiques et sédimentaires : la marée, le débit des cours d'eau qui l'alimentent, les apports sédimentaires. La nature les a façonnés pendant des siècles jusqu'à leur conférer des caractéristiques dont certaines peuvent être reproduites par des lois mathématiques simples. La section mouillée, par exemple, s'accroît d'une façon exponentielle de l'amont vers l'aval. L'estuaire de la Gironde, encore peu aménagé, respecte cette loi, si l'on tient compte de la somme cumulée des sections mouillées de la Garonne et de la Dordogne. La chenalisation de l'estuaire de la Seine, l'approfondissement du chenal de navigation à l'aval, l'extraction des sables et graviers en amont pour l'estuaire de Loire, ont fortement perturbé ces équilibres.

Du point de vue de la biologie, la richesse et la productivité des milieux estuariens ne sont le fait que de quelques espèces, bien adaptées et tirant profit de stratégies spécifiques de reproduction. Des chaînes trophiques courtes permettent à beaucoup d'espèces marines de séjourner pendant certains stades de leur vie. On estime à $65 \%$ le nombre d'espèces de poissons pêchées en Manche qui ont séjourné une partie de leur vie en milieu estuarien.

D'où l'importance, pour sauvegarder ces écosystèmes fragiles, que les aménageurs de l'amont puissent prendre en compte aussi les conséquences de leur ouvrage sur le fonctionnement estuarien.

Dans le rapport $n^{\circ} 1$ de MM. Sanchez, A. Grovel, P. Fattal et Y. Delanoe, les auteurs se proposent, sur des bases bibliographiques, de mettre en évidence quelques aspects sédimentologiques de l'estuaire de la Loire. Après avoir établi les conditions amenant à l'existence d'un bouchon vaseux en estuaire de Loire, les auteurs, sur la base de l'écoulement résiduel, sont amenés à redéfinir l'existence d'un point nodal et à établir une différence entre l'existence d'une surface de vitesse résiduelle nulle et une surface de séparation d'écoulement résiduel. L'existence du bouchon vaseux est expliquée par la présence d'un écoulement à double sens. Ils rappellent aussi les principaux mécanismes qui contrôlent le transport sédimentaire : floculation - défloculation, dépôt, tassement, érosion, déplacements.

Sur la base d'une campagne de mesures en juin 94 , les auteurs s'attachent à définir l'emplacement de la crème de vase et son évolution sur un cycle de marée. De même, à l'aide d'un dispositif permettant d'échantillonner la crème de vase sur la verticale, les auteurs cherchent à mettre en évidence une distribution particulière des concentrations turbides sans y parvenir.

Enfin, une série de carottages et de prélèvements de surface permet d'avancer l'hypothèse que l'apparition des sables au sein de l'estuaire se déclenche au-delà d'un débit de $500 \mathrm{~m} 3 / \mathrm{s}$. Ils concluent que jamais aucun modèle mathématique ne sera capable de reproduire I'ensemble des phénomènes sédimentaires qui se produisent dans un estuaire.

P. Bassoulet et P. Le Hir, dans le rapport $n^{\circ} 2$, examinent l'impact que peut avoir l'élévation du niveau de la mer sur le déplacement du maximum de turbidité dans l'estuaire de Loire. Pour en apprécier les conséquences, les auteurs utilisent un modèle mathématique hydrosédimentaire simplifié.

Il est rappelé ici que les prévisions de l'IPCC retiennent comme possible une augmentation du niveau de la mer allant de 0,3 à $1 \mathrm{~m}$ d'ici 100 ans.

Après avoir établi que le principal mécanisme à l'origine du bouchon vaseux en Loire réside dans la dynamique de la marée, lié particulièrement à la déformation de sa propagation, les auteurs exposent les résultats essentiels produits par le modèle : restitution des hauteurs d'eau, des courants, 
situation et évolution du bouchon vaseux et de la crème de vase.

En application au problème étudié, pour $0,6 \mathrm{~m}$ d'élévation du niveau de la mer (traduit par une modification des conditions aux limites aval), le bouchon vaseux se déplacerait pour un débit d'étiage, de $2 \mathrm{~km}$ vers l'amont. Ce déplacement pourrait être compensé par une augmentation faible des débits. Plus important en terme de gestion, le modèle prévoit l'apparition d'un dépôt de crème de vase renforcée dans la zone de Nantes.

\section{II — TRANSPORT SÉDIMENTAIRE ET ÉRO- SION LITTORALE}

Augmentation du niveau de la mer et déficit dans les apports sédimentaires des fleuves sont les deux raisons les plus importantes évoquées pour expliquer le problème tout à fait actuel de l'érosion côtière. Ces problèmes viennent souvent se superposer et aggraver les évolutions naturelles préexistantes. Plusieurs colloques ont été organisés ces dernières années par la SHF sur ce sujet, il n'est donc pas nécessaire de faire à nouveau un point exhaustif sur cette question. Il est néanmoins intéressant de présenter quelques nouveaux cas, bien documentés, pour évoquer le problème et mettre en évidence les nouvelles techniques d'approche. Quatre communications sont consacrées plus ou moins directement aux problèmes d'érosion côtière.

Deux communications mettent en avant l'utilisation de techniques spécifiques pour évaluer l'origine (sédimentologie, minéralogie) ou le comportement (traceurs radioactifs) des particules non cohésives sur des sites présentant des problèmes importants d'érosion côtière.

Dans les rapports $n^{\circ} 3$ et 4 , les Dr P. Yace, C. Sombo Boko et B. Digbehi Zeli s'engagent sur une recherche des causes de l'érosion des plages du littoral en Côte d'Ivoire.

Dans leur premier document, les auteurs signalent l'existence d'une érosion côtière importante sur le littoral ivoirien $(0,6$ à $1.5 \mathrm{~m} / \mathrm{an})$. Cette érosion est attribuable à un déficit d'apport sédimentaire et les auteurs signalent que celui-ci s'accroît d'ouest en est dans le sens de la dérive littorale. Pour tenter de situer le phénomène, et relier ce déficit à la construction de barrages sur les fleuves Sassandra et Bandama, les auteurs utilisent la sédimentologie et la minéralogie comme outils de pistage des sources sédimentaires pour le littoral. Ainsi des échantillons sont prélevés dans ces barrages, le lit des cours d'eau, les plages du littoral.

Ils concluent, sans le quantifier, à trois origines possibles pour les sédiments des plages : les dépôts issus de la dégradation de l'arrière-pays par les divers facteurs érosifs et drainés par les fleuves, les dépôts issus du remaniement d'anciens cordons littoraux sableux holocènes et les apports provenant du plateau continental par les effets conjugués de la houle et des courants de translation.

Dans leur deuxième contribution, les mêmes auteurs s'attachent à étudier une zone plus restreinte du même littoral, à partir de 29 profils de plage réalisés en vives eaux et à basse mer. Des échantillons sont prélevés en vue d'études granulométriques.
Par comparaison avec des profils relevés à d'autres époques, interprétés en fonction de l'histoire géologique récente (évolution du niveau de la mer), de la houle, de la courantologie locale, de la sédimentologie, les auteurs classent la région en trois zones :

- une zone occidentale «stable» soumise à une érosion saisonnière liée à l'hivernage, aux tempêtes et réalimentée par la dérive littorale ;

- une zone centrale, en érosion continue, et attribuable à l'interruption du transit sédimentaire par trois ouvrages plus ou moins récents : le réservoir ouest (1943), l'ouverture du canal à la Lagune Ebrié (1950), la construction d'une digue d'arrêt des sables :

- une zone orientale en stabilité relative, où le Canyon du «trou-sans-fond» récupère une partie du sable.

Parmi les solutions envisagées par les auteurs pour limiter les problèmes d'érosion, il est proposé des mesures suspensatoires (arrêt des extractions, interdiction de construire), ou bien un rechargement par «clapage» en arrière des plages érodées.

Enfin, il est souhaité que des études plus complètes puissent être réalisées sur le site.

P. Brisset, R. Hoslin et A. Poggi, dans le 5ème rapport, s'attachent à présenter les résultats obtenus lors d'une expérience de marquage radioactif de sables, effectuée sur l'estran des côtes de l'ouest Cotentin. Cette technique éprouvée, utilisée souvent dans le cas d'études liées au transport sédimentaire, a été employée pour évaluer le transport sédimentaire en zone intertidale. De l'ordre de $500 \mathrm{~g}$, de grains de verre contenant de l'iridium ( $\mathrm{Sr} 192,74$ jours de période), de même classe granulométrique que lẹs milieux étudiés, ont été déposés simultanément sur six sites localisés de part et d'autre du Havre de Lessay. Leur suivi dans le temps par sonde nucléaire a permis de suivre leur dispersion, voire leur enfouissement selon le site étudié. Comme principal résultat. les auteurs évoquent des mouvements essentiellement longitudinaux, avec des débits solides faibles, de l'ordre de 0,01 à $1,5 \mathrm{~m}^{3} / \mathrm{m} /$ jour.

Deux autres communications s'attachent à identifier les problèmes d'érosion littorale et à proposer des solutions réalistes.

F. Gazelle présente dans le rapport $n^{\circ} 6$ une étude très complète de l'érosion marine sur le littoral Biarrot. Sur la base de dix années d'observation et au moyen de quelques expériences de marquage à la fluoresceïne, de la mise en place de repères, de levés topographiques, de mesures granulométriques, avec une très bonne connaissance des modifications introduites par l'homme (avec exploitation de documents photographiques anciens), l'auteur nous apporte une vision qui se veut exhaustive de la situation sédimentologique de la zone et nous amène à comprendre les raisons pour lesquelles certaines de ces plages et falaises ont subi au cours du temps des modifications considérables.

L'enchevêtrement de secteurs rocheux et sableux, la présence de hauts fonds et de faibles courants, sont évoqués comme des facteurs de stabilité. L'instabilité est attribuable par l'auteur à deux phénomènes : 
- Un déficit sédimentaire chronique expliqué par la conjonction de phénomènes d'origine naturelle et anthropique. Un changement de la direction de la côte au niveau de l'Adour interfère avec la direction N-S de la dérive littorale dans cette région. Il en résulte une diminution naturelle des apports sédimentaires côtiers, détournée au profit de sables dunaires qui se déplacent vers l'intérieur des terres à raison de $30 \mathrm{~m}^{3}$ par mètre linéaire et par an. Des décennies de prélèvements de sables et graviers, la construction de la grande jetée de l'Adour dans les années soixante, les dragages systématiques à l'embouchure de l'estuaire, viennent s'ajouter aux phénomènes naturels.

- Le recul des falaises, processus général et inéluctable, a été l'objet de travaux de consolidation depuis plus d'une centaine d'années. L'auteur constate que ce recul se poursuit, mais il n'est pas homogène. Il signale par ailleurs que depuis une soixantaine d'années, le pied des falaises a reculé plus vite que leur partie supérieure... Il s'inquiète que cet état des choses empire au fil du temps si des dispositions ne sont pas prises.

La philosophie de l'auteur sur les remèdes à apporter, se base sur la «nécessité d'associer les forces naturelles et les aménagements plutôt que de tenter de contraindre les premières...». Dans cet esprit, il propose la défense du pied des falaises et en diminuer leur verticalité. De même, il estime nécessaire la mise en place d'épis surbaissés perpendiculaires à la houle et au courant, et de relier entre eux le bas des Jumeaux d'Ilbarritz et de les «souder « au continent.

Dans leur rapport $n^{\circ} 7$, J. Viguier et B. Bellesort, dans le cadre d'une demande exprimée par le Parc Régional Naturel de Camargue, analysent l'évolution des côtes sur le littoral camarguais et proposent une stratégie d'aménagement. Après avoir passé en revue les différentes données d'origine bibliographique, les auteurs ont procédé à un ensemble de campagnes sur le terrain pour acquérir des données bathymétriques, topographiques et écologiques, situer les aménagements existants et établir une synthèse des principales activités humaines sur le littoral.

Parmi les facteurs d'évolution du littoral, les auteurs en identifient trois : l'action du vent sur les dunes dégradées, l'agitation du milieu marin qui entraîne le «rabotage» des anciens deltas et modifie le transit littoral, et enfin l'évolution «naturelle» du delta du Rhône, avec la migration en 1711 du bras principal vers sa situation actuelle.

Récemment, pour freiner cette évolution et réalimenter les plages, 105 épis perpendiculaires à la côte ont été construits sur environ $30 \mathrm{~km}$, c'est-à-dire la moitié du littoral camarguais. Les effets espérés sont, semble-t-il décevants, à cause du transit littoral relativement limité et des estrans à faible pente peu favorables au piégeage des sédiments par des épis courts.

Par contre, les aménagements effectués en milieu terrestre (protection des pieds des dunes, piégeage des sables par des ouvrages longitudinaux) semblent avoir été efficaces.

Un classement sur la vulnérabilité du littoral de Camargue est alors proposé par les auteurs sur la base des problèmes d'érosion actuelle, de l'usage qui est fait de la zone littorale (tourisme, pêche, industrie) et de la réglementation en vigueur. Puis ils définissent une stratégie d'aménagement en tenant compte d'une éventuelle remontée de la mer, en proposant trois scénarios : intervention minimale, maintien de la situation actuelle, amélioration de la situation actuelle. En particulier, ils recommandent de favoriser les solutions de «rechargements», notamment avec des galets, qui apportent le moins de perturbations à l'environnement.

\section{III — LES PROCESSUS SÉDIMENTAIRES}

La qualité de la modélisation numérique en hydrodynamique et transport sédimentaire (matériel cohésif on non) en milieu côtier, et leur aptitude à traiter des problèmes de plus en plus complexes dépendent de plusieurs facteurs : acquérir de bonnes données pour le calage et/ou la calibration, disposer d'une connaissance solide sur les processus à modéliser, avoir une bonne quantification de ceux-ci, et enfin, posséder des codes de calcul performants, l'ensemble de ces éléments constituant le «savoir faire» d'un chercheur ou d'un bureau d'études.

S'il est un domaine dans lequel les connaissances sur les processus sont toujours nécessaires, c'est bien la sédimentologie.

Dans le rapport $\mathrm{n}^{\circ} 8$ portant sur l'interaction houle-courant-sédiment, T. Tran Thu et A. Temperville exposent les différents termes qui interviennent dans la modélisation du transport en suspension de sédiments non-cohésifs sous l'action d'un écoulement de type houle plus courant. Un modèle numérique $1 \mathrm{DV}$ de fermeture K-L est appliqué aux résultats d'expériences en laboratoire réalisées dans le cadre du programme MAST2/G8M. II fournit la distribution verticale de la concentration en MES, leur variation temporelle ainsi que le débit solide.

Après avoir montré que les distributions spatiale et temporelle des concentrations étaient qualitativement bien reproduites, que la concentration moyenne et les quantités intégrées étaient satisfaisantes, les auteurs attribuent les erreurs de phase de la concentration et des quantités dérivées de la concentration instantanée à la non prise en compte dans le modèle, de l'échange intergranulaire de quantité de mouvement dans la couche de transport par charriage.

Ils concluent en estimant que ce type de modèle peut permettre la paramétrisation du débit solide de façon à être introduit dans les applications de génie côtier.

Un domaine sur lequel l'ensemble des sédimentologues s'accorde volontiers, est le manque de données sur la vitesse de chute réelle des particules, en particulier celles à comportement cohésif. Paramètre essentiel dans la modélisation mathématique du transport sédimentaire, cette information est difficilement accessible par l'expérimentation, qu'elle soit en laboratoire (difficulté de reproduire la turbulence et les processus de floculation - défloculation) ou «in situ» (problèmes dus à l" «échantillonnage»).

Dans le rapport $n^{\circ} 9, \mathrm{~S}$. Cadiergue, N. Michaux-Leblond et $M$. Belorgey, se proposent de mettre en évidence l'influence des structures turbulentes sur la vitesse de chute de particules solides. A l'aide d'un dispositif expérimental 
constitué d'une veine d'essai verticale couplée à une grille génératrice de turbulence, les auteurs s'attachent à quantifier la vitesse de chute de particules solides de diamètre compris entre 2.5 et $4 \mathrm{~mm}$ et constituées de verre, agate et acier.

Les résultats expérimentaux laissent apparaitre un ralentissement de la chute des particules engendré par les structures turbulentes. S'il est vrai que la taille des particules utilisées lors des expériences n est pas tout-à-fait dans la gamme de celles rencontrées couramment dans le milieu côtier. le dispositif expérimental semble opérationnel et applicable à d'autres types de particules. On peut espérer que des tentatives analogues pourront être faites sur des particules qui floculent dans un milieu turbulent.

\section{IV a LES OUTILS DE MODÉLISATION EN HYDRODYNAMIQUE ET TRANSPORT SÉDIMENTAIRE : CAS D'APPLICATION}

Sont présentés ici un ensemble de rapports qui ont comme point commun l'application de la modélisation numérique à des problèmes d'applications destinées à l'aménagement. D'abord, deux communications sur de problèmes d'hydrodynamique, puis deux autres sur des problèmes liés au transport sédimentaire.

Dans leur rapport $n^{\circ} 10$, P. Sauvaget et $E$. David exposent une étude numérique du fonctionnement hydrodynamique d'un plan d'eau côtier profond méditerranéen, et son application à la Baie de Monaco.

La modélisation côtière en Méditerranée en est à ses débuts. Dissipation de l'énergie de la houle et du courant. apports d'eau douce, hydrodynamique largement induite par l'interaction du courant liguro-provençal avec les irrégularités de la côte et de la bathymétrie, conditions hydro-climatiques particulières, stratification haline et thermique : la complexité du fonctionnement des systèmes méditerranéens, on le voit, est incontestable.

Les auteurs, après avoir rappelé les principaux forçages de la circulation côtière en Méditerranée. ont choisi la baie de Monaco, comme site d'application pour la modélisation hydrodynamique. Dans le cadre d'un contrat européen (MEDCOST) quatre codes de calcul différents ont été utilisés. Compte tenu des conditions baroclines, les auteurs présentent ici les résultats obtenus à l'aide du code TELEMAC-3D (tridimensionnel). Les conditions initiales et aux limites sont issues des données de température et de salinité sur la verticale obtenues dans le cadre de la campagne PRIMO-0. Un calcul géostrophique a permis d'évaluer le champ initial des vitesses. La surface libre est déclarée horizontale à l'instant initial, elle peut évoluer librement pendant le calcul, sauf sur la frontière maritime parallèle à la côte où elle est imposée à zéro.

Le modèle a été testé sur un certain nombre de combinaisons liées aux forçages : circulation induite par le courant Ligure en conditions hivernale et estivale. forçage par un coup de vent variable et homogène sur l'ensemble du domaine, effet des apports d'eau douce d'origine continentale en surface. Par ce travail, les auteurs mettent en évi- dence la présence d'un vortex en face de Cap Ferrat, d'un contre-courant très côtier, d'une remontée des eaux profondes sous l'effet du vent et de l'étalement en surface de la nappe d'eau douce.

Les auteurs concluent à l'intérêt de disposer d'une telle approche pour la gestion du milieu marin en Méditerranée et affichent le besoin de disposer de données locales de bonne qualité.

J.L. Rahuel, M. Erlich et P. Chaillet présentent, dans le cadre du rapport $\mathrm{n}^{\circ} 11$ un autre exemple de l'utilisation de la modélisation mathématique pour effectuer une prévision opérationnelle des inondations de la ville de Shanghaï. En effet, cette ville de 13 millions d'habitants, construite sur le delta du Yangtzé, est menacée périodiquement d'inondations du fait de la conjonction de trois facteurs : son niveau est plus bas que le niveau des marées moyennes ; la région est soumise à de fortes précipitations augmentant fortement le débit des rivières. De plus, ceci se produit souvent en association avec une élévation du niveau de la mer, conséquence du passage des cyclones.

Une chaîne de prévision complète contre les inondations a donc été mise en place dans le cadre d'une coopération entre I'Union Européenne et la République Populaire de Chine. Ce système met en æuvre une chaîne de simulations en temps réel comportant les éléments suivants :

- un réseau télématique en différents points du réseau hydrographique mesurant et transmettant les variables hydrométéorologiques sensibles.

- le modèle PLUDEB de type boite noire transforme les informations concernant la pluviométrie en débits (en réalité volume produit),

- un modèle 2D horizontal de marée-tempête, qui, sur la base de données fournies sur la marée astronomique et les caractéristiques du typhon, permet de calculer à tout moment le niveau de la mer.

- enfin. le modèle hydrodynamique CARIMA/CASCADE qui, à partir des informations produites par les autres étapes du système, calcule débits et vitesses dans l'ensemble hydrographique du delta.

Les auteurs font part de leur souci permanent concernant les besoins de robustesse et de fiabilité de la chaîne de prévision dans le contexte d'une simulation en temps réel. Chaque élément de la chaîne est ainsi testé indépendamment. A tout moment le système doit trouver des informations même partiellement dégradées. Toute disposition est prise pour que le calcul puisse démarrer à tout moment sur un état initial le plus proche possible de la réalité. Ce système, perfectible dans le temps, opérationnel depuis janvier 1996. exécute la chaîne de prévisions en quelques minutes. Les auteurs estiment qu'il est encore trop tôt pour tirer des conclusions définitives, mais signalent que les résultats préliminaires sont encourageants et valident la justesse des choix opérés en termes de modélisation. Un travail de valorisation des données historiques resterait à effectuer par la partie chinoise.

La communication $n^{\circ} 12$, proposée par R. Silva Jacinto et P. Le Hir, porte sur une étude méthodologique de la simulation des agitations dans l'embouchure de la Seine. 
En estuaire de Seine, les processus sédimentaires jouent un grand rôle, soit au sein du système bouchon vaseux - crème de vase, soit au niveau des vasières intertidales et des zones humides. La modélisation des structures turbides, un des objectif définis dans le cadre du programme Seine Aval, nécessite de pouvoir reproduire les agitations (houles et clapots) à l'embouchure.

Pour développer une méthodologie adaptée, les auteurs font appel au modèle de houle HISWA développé par la TUDelft, qui résout l'équation de conservation de densité d'action de houle en conditions stationnaires de vent et de courant.. Ce modèle est utilisé sur deux échelles géographiques :

- la Baie de Seine (voire une partie de la Manche) en négligeant les courants et la variation des niveaux d'eau,

- l'embouchure de la Seine, où les effets de la marée ont été pris en compte à l'aide du modèle SAM-2DH.

Pour assurer la pertinence des résultats obtenus, les auteurs ont procédé à une analyse de sensibilité de certains paramètres dont l'étalement directionnel de la houle incidente et le coefficient de frottement lié à la houle. Le modèle s'est montré robuste, avec des différences dans le secteur étudié toujours inférieures à $5 \%$. En revanche, il est démontré en terme de vitesse orbitale au fond, l'absolue nécessité d'avoir une bonne connaissance de la rugosité du fond.

La méthodologie a été appliquée à un clapot généré par un vent d'ouest de force 4 auquel a été superposée une houle d'ouest, de hauteur significative $1 \mathrm{~m}$ et de période moyenne de 10 s. Après une courte discussion sur les résultats obtenus, les auteurs confirment l'importance de l'effet du courant (réfraction) sur les conditions d'agitation dans l'embouchure de la Seine. Un travail de validation reste encore à faire dans les zones de déferlement, où les connaissances des vitesses orbitales ne sont plus suffisantes pour la remise en suspension des particules.

Toujours dans le domaine d'application des outils numériques aux problèmes de génie côtier, J.M. Tanguy traite dans le rapport $\mathrm{n}^{\circ} 13$, de trois problèmes d'application. Auparavant, l'auteur brosse un panorama complet des techniques de modélisation numérique applicables aux zones littorales, en soulignant les différentes avancées technologiques (informatique, réseaux de communication, capacités graphiques) et scientifiques (modélisation numérique, meilleure connaissance des processus physiques) permettant maintenant d'obtenir d'excellents rendements de ces outils en termes opérationnels.

Il souligne enfin l'intérêt qu'ont les différentes équipes à s'associer dans le cadre de programmes européens afin de réduire les coûts et augmenter la capacité d'expertise.

Parmi les différents cas d'application proposés dans ce rapport, J.M. Tanguy aborde la situation du Port d'Antifer qui se trouve confronté à deux problèmes : la présence de forts courants en certains moments de la marée, qui gênent l'entrée du port pétrolier, ainsi que l'apparition à proximité du musoir de la digue, d'une importante fosse relevée lors de récents sondages bathymétriques.

D'abord à l'aide du modèle REFLUX (modèle de courantologie 2D), l'auteur a calculé la hauteur d'eau et le champ vitesse dans deux domaines «emboîtés», en utilisant comme conditions aux limites les résultats fournis par le SHOM qui dispose d'un modèle bien calibré de la Manche. Les calculs montrent clairement l'apparition de courants dépassant $2 \mathrm{~m} / \mathrm{s}$ au droit du musoir avant la pleine mer.

Puis à l'aide de modèle SISYPHE (transport des sédiments non-cohésifs), couplé à REFLUX, l'auteur met en évidence une érosion naturelle de la zone incriminée, prédominante en vives eaux, et du même ordre de grandeur que celle trouvée par les sondages (environ $1 \mathrm{~ms} / \mathrm{an}$ ).

Deux autres applications sont présentées par l'auteur, l'une au Ghana où il s'agissait de simuler le comportement morphologique de l'embouchure d'un chenal reliant un lagon à la mer, l'autre, plus «théorique», traitant de la simulation de l'évolution des fonds d'une plage autour d'un brise-lames avec une comparaison des résultats avec des essais conduits par SOGREAH dans un canal à houle. Pour ces deux exemples encore, l'utilisation de la panoplie de modèles tels que VAG (réfraction de houle) REFLUX, SISYPHE, a conduit à des résultats satisfaisants. Cela conduit l'auteur à conclure en faisant le point sur les problèmes rencontrés au cours de la modélisation (le problème du maillage, l'interpolation des fonds, l'obtention de bonnes conditions aux limites) et à encourager le développement de systèmes de modélisation intégrés faisant appel à une batterie de codes homogènes.

\section{V a MODÉLISATION EN QUALITÉ DES EAUX}

Les possibilités offertes par la modélisation mathématique sont très inégales selon le niveau de complexité des problèmes à traiter. Comme nous venons de le voir, l'hydrodynamique et dernièrement le transport sédimentaire sont des étapes qui sont de plus en plus à la portée de tout bon laboratoire de recherche. Nous avons néanmoins perçu que beaucoup d'auteurs manquent encore cruellement de données de terrain. soit pour caler les modèles, soit pour affiner les processus hydrosédimentaires (vitesses de chute de particule, tassement, liquéfaction des vases, etc.).

Dans le domaine de la qualité des eaux, il reste beaucoup plus à faire. La modélisation des éléments naturels est en bonne voie ; productions primaire et secondaire, cycle du carbone et de l'azote, activité microbiologique autotrophe ou hétérotrophe, cycle de l'oxygène, sont des variables que l'on trouve de plus en plus modélisées dans des systèmes estuariens ou côtiers. Ce n'est plus ici la capacité de calcul qui est en cause, mais bien la connaissance et la quantification des processus élémentaires. Celles-ci se font généralement en laboratoire sur des échantillons prélevés dans les milieux à modéliser. De même, l'interfaçage entre modèles hydrodynamiques, modèles de transport sédimentaire et modèles de qualité des eaux sont confrontés à des problèmes d'hétérogénéités temporelles ou spatiales non négligeables.

La modélisation des contaminants en milieu côtier est aux premiers stades de développement. Cette démarche est tributaire d'une part du bon fonctionnement de l'ensemble des autres disciplines, toutes fournissant les informations nécessaires pour soutenir une bonne approche et d'autre part, la complexité des phénomènes bio-géo-chimiques nécessite des formulations que l'on est loin de savoir maîtriser. 
Un bon exemple des démarches entreprises dans ce thème et des quelques problèmes rencontrés est fournie par la contribution $\mathrm{n}^{\circ} 14$ (J.L. Gonzalez et B. Thouvenin) sur les apports de la modélisation à la compréhension du comportement du cadmium en milieu estuarien.

Les auteurs préfèrent pour le moment utiliser la modélisation comme un outil explicatif plutôt que prédictif. En particulier, ils cherchent à quantifier, à partir de données obtenues expérimentalement en laboratoire, des paramètres qui ne sont pas mesurables directement sur le terrain. De même. le modèle doit leur permettre d'évaluer l'importance sur le cycle du métal, de certains paramètres spécifiques tels que concentration en ligands, nature minéralogique des particules, propriétés de surface, etc.

Ce modèle «chimique», développé et appliqué pour le cadmium, tente de décrire le comportement (échanges dissous-particulaire) de cet élément en milieu côtier. Il permet de reproduire numériquement la spéciation du cadmium, en prenant en compte différentes variables environnementales importantes pour la distribution de ce contaminant (salinité, $\mathrm{pH}$, ligands dissous, concentration et nature des particules...). Ce type de modèle traite toutes les réactions homogènes (formation de complexes dissous) ou hétérogènes (adsorption/désorption) comme des réactions de complexation.

L'exemple présenté montre les limites de cette approche qui, pour être opérationnelle, doit se baser sur la connaissance de nombreux paramètres mesurés sur le terrain et en laboratoire. De plus, l'application de cet outil à un site donné requiert un couplage à des modèles hydrosédimentaires qui reproduisent avec une bonne précision la distribution spatio-temporelle de toutes les variables nécessaires aux calculs de spéciation.

La qualité des eaux côtières en termes de risques sanitaires est évaluée par le comptage de bactéries entériques prises en tant que témoin de pollution fécale. Celles-ci sont apportées au milieu marin par les rivières et divers émissaires et égouts. Leur nombre important peut conduire à mal classer une plage, voire la fermer. Les plages de Mers-lesBains, Eu et le Tréport présentent une situation qualitative médiocre et précaire, ce qui a conduit les administrations responsables de l'assainissement à entreprendre une étude exhaustive des différentes sources de contamination (diagnostic des réseaux de collecte et des stations d'épuration), ainsi que l'étude des différentes voies de contamination des plages. Dans le rapport $\mathrm{n}^{\circ} 15, \mathrm{C}$. Zobrist, L. Herremans, J. Coutelle, J. Auvray et D. Munro, présentent les résultats de cette étude basée sur l'utilisation de modèles de calcul construits autour de logiciels simulant le comportement des germes test. Sans entrer dans le détail des calculs, les auteurs nous renseignent sur la démarche entreprise, basée sur le couplage de codes de calcul de différentes origines. Les apports ont été estimés à l'aide du logiciel FLUPOL sur la base des mesures effectuées sur le terrain. Le modèle bactériologique appliqué pour évaluer l'impact de ces apports est celui bâti par le WRc. Celui-ci s'appuie pour I'hydrodynamique en mer sur un module courantologique développé par le $\mathrm{LNH}$ pour les besoins de la centrale nucléaire de Penly toute proche, en rivière de la Bresle sur un module de transport simple (unidimensionnel).
A partir d'objectifs d'assainissement pré-définis, les auteurs ont testé différentes hypothèses de travail par temps sec ou par temps de pluie. Après avoir identifié que le problème était attribuable aux apports en période de pluie, les auteurs se sont attachés à tester les effets d'un stockage temporaire de ces apports dans des bassins. Ils concluent néanmoins que pour obtenir une maîtrise durable de la qualité des plages, il est nécessaire de supprimer tout rejet d'eau brute non traitée

\section{VI $\square$ CONCLUSION}

Ce n'est pas avec les quelques exemples présentés que nous pouvons brosser un panorama complet des problèmes qui se posent sur le littoral. Nous constatons la place dominante prise dans ce thème par les problèmes liés à l'hydrodynamique et au transport sédimentaire. Mais nous avons aussi constaté la place importante tenue par ces disciplines dans les approches environnementales. Nous avons aussi constaté que les actions à entreprendre pour parvenir à une bonne gestion du milieu estuarien et littoral sont le plus souvent pluridisciplinaires et demandent la mise sur pied de programmes parfaitement coordonnés. Déjà, en milieu estuarien, des approches pluridisciplinaires de modélisation voient le jour en Europe et en France. Nous en reparlerons au cours des prochaines journées de la SHF consacrées au milieu marin.

Des outils de calcul performants commencent à se mettre en place. La modélisation du transport sédimentaire, qu'elle s'adresse à du matériel cohésif ou non, est en bonne voie. mais le manque de données expérimentales sur le terrain, difficiles à obtenir, et mobilisant des sommes importantes, est actuellement un handicap. L'ouverture que nous avons constatée dans ce thème vers les autres laboratoires du continent dans le cadre des appels d'offres issus de l'Union européenne, témoigne d'une recherche de synergie qui est tout à fait encourageante. Elle ne peut être que profitable à tous.

Dans les problèmes de transport sédimentaire et d'érosion côtière, les études sur l'action simultanée des houles et des courants sur les mouvements sédimentaires restent toujours d'actualité. D'autant plus que les effets prévisibles liés à la probable remontée du niveau de la mer ne semblent plus être un cas d'école.

Ayons une pensée pour la Méditerranée où la complexité de la situation hydroclimatique rend difficile l'approche par modélisation en zone côtière. Des efforts sont en cours et doivent être également encouragés.

Pour revenir au thème de ces Journées, «Les chemins de l'Eau», il est de plus en plus évident que les systèmes hydrographiques doivent être traités comme un tout, comme un «continuum» depuis l'amont des bassins versants jusqu'au proche littoral. Le législateur français en a pris conscience dès l'année soixante par la création des Agences de l'Eau. Ces établissements, dotés d'un grand pouvoir incitatif, sont actuellement les seuls à pouvoir intégrer la gestion de l'ensemble du bassin. C'est une collaboration étroite entre scientifiques, Agences de l'Eau, administrations régionales et locales, qui permettra de faire passer, dans les faits, l'application des nouvelles connaissances scientifiques (et leur volet technologique) à la résolution des problèmes de l'eau et de la nature. 